\title{
Fuel Retention Studies with the ITER-like Wall in JET
}

\author{
S. Brezinsek ${ }^{1}$, T. Loarer ${ }^{2}$, V. Phillips ${ }^{1}$, H.G. Esser ${ }^{1}$, S. Grünhagen ${ }^{3}$, R. Smith ${ }^{3}$, R. Felton ${ }^{3}$, \\ J. Banks ${ }^{3}$, P. Belo ${ }^{4}$, A. Boboc ${ }^{3}$, J. Bucalossi ${ }^{2}$, M. Clever ${ }^{1}$, J.W. Coenen ${ }^{1}$, I. Coffey ${ }^{5}$, D. \\ Douai $^{2}$, M. Freisinger ${ }^{2}$, D. Frigione ${ }^{6}$, M. Groth ${ }^{7}$, A. Huber ${ }^{1}$, J. Hobirk ${ }^{8}$, S. Jachmich ${ }^{9}$, \\ S. Knipe ${ }^{3}$, U. Kruezi ${ }^{2}$, G.F. Matthews ${ }^{3}$, A.G. Meigs ${ }^{3}$, F. Nave ${ }^{4}$, I. Nunes ${ }^{4}$, R. Neu ${ }^{8}$, J. \\ Roth $^{8}$, M.F. Stamp ${ }^{3}$, S. Vartagnian ${ }^{2}$, U. Samm ${ }^{1}$ and JET EFDA contributors ${ }^{\star}$
}

JET-EFDA, Culham Science Centre, Abingdon, OX14 3DB, UK

${ }^{1}$ IEK-4, Forschungszentrum Jülich, Association EURATOM-FZJ, Germany

${ }^{2}$ CEA, IRFM, F-13108 Saint-Paul-lez-Durance, France

${ }^{3}$ EURATOM/CCFE Fusion Association, Culham Science Centre, Abingdon, OX143DB, UK

${ }^{4}$ Institute of Plasmas and Nuclear Fusion, Association EURATOM-IST, Lisbon, Portugal

${ }^{5}$ Queen's University Belfast, BT71NN, UK

${ }^{6}$ Associazione EURATOM-ENEA sulla Fusione, CP 65, Frascati, Rome, Italy

${ }^{7}$ Aalto University, Association EURATOM-Tekes, Espoo, Finland

${ }^{8}$ Max-Planck-Institut für Plasmaphysik, EURATOM Association, D-85748 Garching

${ }^{9}$ Association Euratom-Etat Belge, ERM-KMS, Brussels, Belgium

Corresponding Author: sebastijan.brezinsek@jet.efda.org

${ }^{\star}$ See the Appendix of F. Romanelli et al., Proceedings of the 24th IAEA Fusion Energy Conference 2012, San Diego, US

\section{Abstract:}

JET underwent a transformation from a full carbon-dominated tokamak to a full metallic device with the ITER-like wall combination for the activated phase with Beryllium main chamber and Tungsten divertor. The ITER-Like Wall (ILW) experiment at JET provides an ideal test bed for ITER and shall demonstrate as primary goals the plasma compatibility with metallic walls and the reduction in fuel retention. We report on a set of experiments $\left(I_{p}=2.0 M A, B_{t}=2.0 T-2.4 T, \delta=0.2-0.4\right)$ in different confinement and plasma conditions with global gas balance analysis demonstrating a strong reduction of the long term retention rate by a factor ten with respect to carbon references. All experiments have been executed in a series of identical plasma discharges in order to achieve maximum plasma duration until the analysis limit of the active gas handling system has been reached. The composition analysis shows high purity of the recovered gas, typically $99 \%$ D. For typical L-mode discharges $\left(P_{\text {aux }}=0.5 \mathrm{MW}\right)$, type III $\left(P_{\text {aux }}=5.0 \mathrm{MW}\right)$, and type I ELMy H-mode plasmas $\left(P_{\text {aux }}=12.0 \mathrm{MW}\right)$ a drop of the retention rate normalised to the operational time in divertor configuration has been measured from $1.27 \times 10^{21} \mathrm{Ds}^{-1}, 1.37 \times 10^{21} \mathrm{Ds}^{-1}$, and $1.97 \times 10^{21} D s^{-1}$ down to $4.8 \times 10^{19} D s^{-1}, 7.2 \times 10^{19} D s^{-1}$, and $16 \times 10^{19} D s^{-1}$, respectively. The dynamic retention increases in the limiter phase in comparison with CFC, but also the outgassing after the discharge has risen in the same manner and overcompensates this transient retention. Overall an upper limit of the deuterium retention rate of $1.5 \times 10^{20} \mathrm{Ds}^{-1}$ has been obtained with the ILW. The observed reduction by one order of magnitude confirms the expected predictions concerning the plasma-facing material change in ITER and widens the operation without active cleaning in the DT phase. 


\section{Introduction}

The currently proposed ITER start-up material combination for the non-active phase consists of plasma-facing components (PFCs) made of Be for the main chamber wall, Carbon-Fibre Composites (CFC) for the divertor target plates, and $\mathrm{W}$ for the divertor baffle and dome area. An exchange of the divertor to a full $\mathrm{W}$ divertor is foreseen for the activated operational phase [1]. The replacement of CFC by $\mathrm{W}$ is governed by the need to remain within the safety limit for in-vessel tritium inventory, and thus, to minimise the long term fuel retention. The latter is in carbon-dominated machines determined by co-deposition with $\mathrm{C}$ which is transported stepwise to remote areas [2]. The full metallic material combination, Be main chamber and $\mathrm{W}$ divertor, should, according to comprehensive studies described in [3], increase the operational time without active cleaning intervention of more than one order of magnitude with respect to a hypothetical all carbon ITER and about a factor 4-5 to the initial material mix with CFC target plates.

The world largest tokamak JET underwent a transformation from a full carbon-dominated device with all PFCs made of CFC to a full metallic device with the ITER material combination for the activated phase. The replacement of all CFC by bulk and coated Be PFCs in the main chamber, bulk $\mathrm{W}$ at the outer horizontal target plate, and $\mathrm{W}$-coated CFC elsewhere in the divertor was done by remote handling in a single shutdown [4]. The ITER-Like Wall (ILW) experiment at JET, which includes dedicated experimental preparatory work with a set of reference discharges $[5,6]$, provides therefore an ideal test bed for the ITER material choice and shall demonstrate as primary goals the plasma compatibility with the new metallic wall and the expected reduction in fuel retention [7]. Initial experiments have shown the successful operation with metallic walls though with a narrower operational window at the best confinement in the ITER-base line scenario caused by the required operation at higher divertor densities to minimise the $\mathrm{W}$ influx [8]. The absence of CFC as PFC material has led even without active cleaning of the Inconel vessel to a dramatic reduction of the carbon content in the plasma by more than one order of magnitude [9]. The temporal evolution of the $\mathrm{C}$ content in the plasma was documented by optical spectroscopy in monitoring discharges which revealed three phases: an initial clean-up phase, a constant phase, and at the end of campaign a phase with slight increase of $\mathrm{C}$ with auxiliary power [9]. However, the carbon flux in the plasma edge - a key parameter for the long term retention in JET-C - was reduced by at least a factor 10 throughout the first JET-ILW year.

Here, we report on a set of deuterium experiments with gas balance analysis demonstrating a strong reduction of the long term fuel retention with the ILW with respect to previously performed JET-C references under various plasma conditions. These global gas balance experiments have been performed throughout the campaign; starting with ohmic plasmas in the initial phase of divertor operation and finishing in high confinement mode (H-mode) plasmas at the last day of operation. The short term retention -studied in-situincreases in the limiter plasma phase with the Be first wall, but the outgassing after the discharge has risen in the same manner and compensates this transient retention. Overall, the reduction of the long term retention is fully supportive in the ITER material choice and widens the operation in DT without active cleaning. Details about the experimental method are described in sec. 2, experimental results are presented in sec. 3, followed by the discussion and conclusion section 4 addressing the consequences for ITER. 


\section{Experimental Method}

The quantification of the retention rate, i.e. the number of retained deuterium ions in the first wall components per second, has been performed by global gas balance in the JET vessel. The gas balance takes into account the amount of injected D through the gas injection systems and, if applicable, the neutral beam injection, and the number of actively pumped neutrals by the applied pumping systems. The pumped gas is collected by the JET active gas handling system (AGHS), quantified by calibrated pressure measurements, and analysed for its components by gas chromatography; the quantification is currently limited to $2600 \mathrm{Pam}^{3}$. The internal precision of AGHS concerning the quantification and composition is $1.0 \%$ [10]. All data presented here are normalised to $293.15 \mathrm{~K}$.

From the technical point of view three types of gas balance experiments were performed whereas the letters (a-e) of the specific experiment in either ohmic, L or H-mode confinement regime correspond to the columns in fig. 1 and the text below:
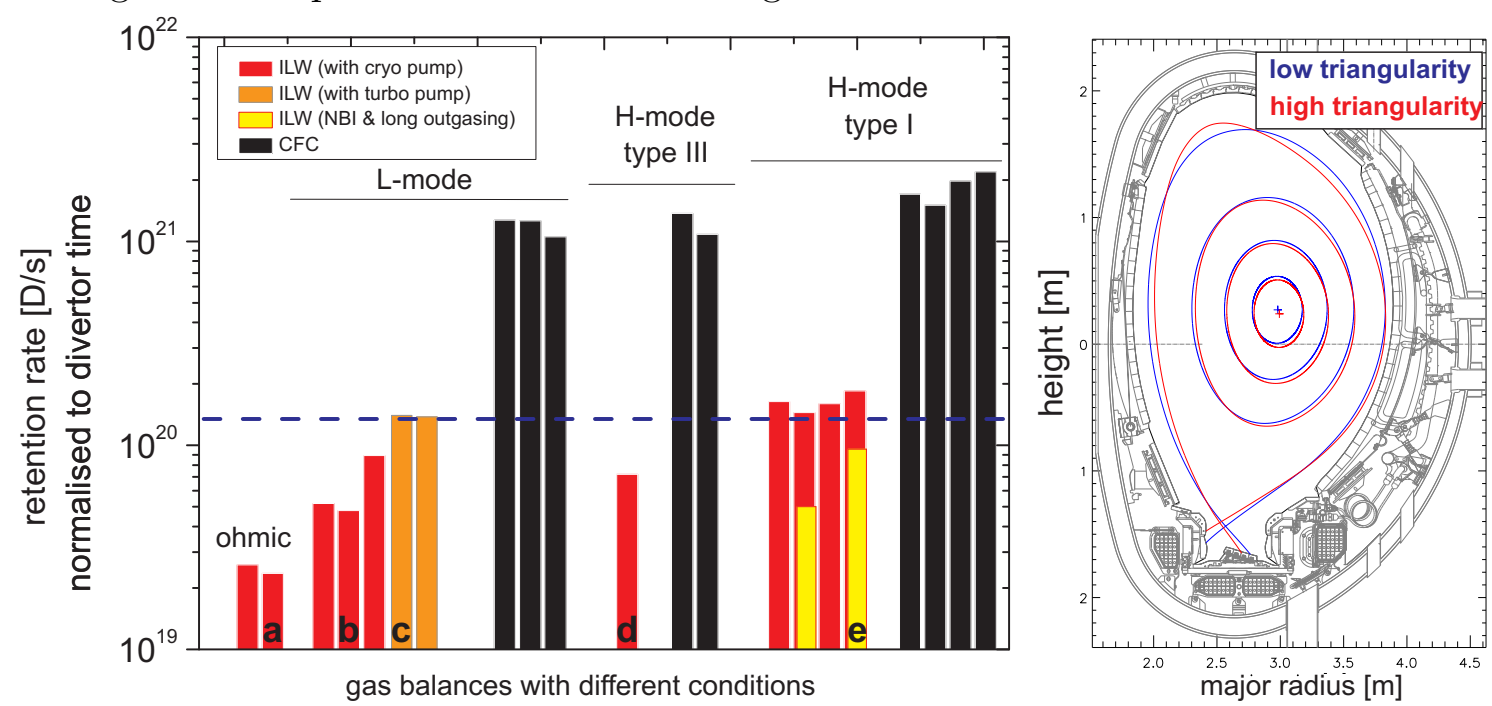

FIG. 1: a) Measured D retention rates (logarithmic scale) for different plasma and confinement conditions in JET with the ILW and related to JET-C references. b) Plasma configurations covered in the JET-ILW experiments and references.

(i) Active use of the cryogenic divertor pump at LHe (liquid Helium) temperature and no active pumping by turbo-molecular pumps (a-b). The cryogenic pump is regenerated before and after the experiment which consists of a number of repetitive comparable discharge. The gas collected by AGHS after the second regeneration represents the actively pumped D during plasma operation and in-between discharges by outgassing. The vacuum valve to the neutral beam cryogenic pump is closed in this standard method. This method had been used before in JET-C [6].

(ii) Active pumping of the neutrals solely by the turbo-molecular pumps whereas all cryogenic pumps are inactive and stay at LN temperature (c). The exhaust from the turbo-molecular pumps is collected and analysed by AGHS as described before. This decreases the gas throughput per plasma discharge, which increases the statistics and the precision of the gas balance by about a factor 4 , but reduces the pumping capability in the ramp-down phase and making the discharges somewhat more prone to minor density limit disruptions. Here, only experiments in L-mode were carried out which show a slightly higher retention rate with respect to (i). 
(iii) Active use of the cryogenic divertor pump at LHe temperature as described in (i) but with additional pumping of the vessel by the neutral beam cryogenic system (d-e). The plasma operation with auxiliary heating by the neutral-beam system, i.e. H-mode plasmas, requires use of the NBI cryogenic pump system. In this operation mode the fraction of gas actively pumped by the NBI system was deduced from the ratio of pumping speeds of the NBI versus the divertor cryogenic pump system. The pumping speeds have been measured in-situ in dedicated gas test pulses at the start of each gas balance experiment resulting in $76 \mathrm{~m}^{3} \mathrm{~s}^{-1}$ for the NBI system in comparison with $118 \mathrm{~m}^{3} \mathrm{~s}^{-1}$ for the divertor cryogenic pump system with respect to the neutral pressure measured in the JET vessel. The major impact of the NBI cryogenic pumps is in the post discharge outgassing in which the system contributes to about $1 / 3$ of pumped neutrals.

The overall precision of the gas collection, including gas injection, transfer lines, and quantification in AGHS has been determined in calibration experiments without plasma which mimic the injection conditions with plasma. The first experiments $[(\mathrm{a}),(\mathrm{b})]$ revealed a reproducible systematic uncertainty in the temperature normalisation in AGHS which lead to an overestimation of the gas loss, therefore, the calibration value has been subtracted from the actual measurement with plasma to provide the retention rate. The uncertainty is given by the statistical error of the reproducibility of the calibration. An upgrade of the AGHS has been used in experiments [(c)-(e)] providing an uncertainty of the overall method of $\pm 1.2 \%$ which has been verified throughout the campaign in calibration experiments performed temporarily close to the actual plasma experiment.

\section{Experimental Results}

From a set of JET-ILW global gas balances which are described in fig.1 in the order of temporal appearance during the campaign, we present here in detail five dedicated experiments (a-e) executed at a plasma current of $I_{p}=2 M A$, a toroidal magnetic field of either $B_{t}=2.0 T$ or $2.4 T$ with a triangularity of $\delta=0.2$ and $\delta=0.4$, respectively, covering different plasma conditions, confinement regimes, and injection rates. The corresponding plasma shapes in high and low triangularity are shown in fig. 1b). All experiments were carried out in series of comparable repetitive discharges, minimum 9 and maximum 34 consecutive plasmas, until the number of injected particles reaches approximately the analysis limit of the AGHS which minimises the impact of history effects and maximise the plasma exposure. An overview of all measured deuterium retention rates in JET-ILW experiments and corresponding JET-C references is given in fig. 1. The retention rates are normalised to the integrated plasma time in divertor configuration reflecting the plasma time with main ion flux interaction with the divertor PFCs. The ILW discharges have been adapted in input power, magnetic configuration and fuelling rate to match edge plasma conditions, i.e. the density and deuterium ion flux, of CFC references as close as possible. However, it should be noted that ILW discharges are optimised with respect to the power loads on the Be limiters and therefore the discharge start-up in limiter configuration is reduced by $\approx 5 s$ in comparison with the references. Apart from the material comparison, the fuelling rate $\left(1 \times 10^{21}-1 \times 10^{23} \mathrm{Ds}^{-1}\right)$, also the auxiliary heating $\left(P_{a u x}=0-12.0 \mathrm{MW}\right)$, as well as the perpendicular ion flux to the divertor $\left(\Gamma_{i o n}=2 \times 10^{22}-2 \times 10^{23} m^{2} s^{-1}\right)$ has been varied. All JET-ILW experiments show low retention rates covering a range of $2-16 \times 10^{19} D s^{-1}$ applying the same analysis as in JET-C and show high purity of the recovered gas of more than $99.0 \% \mathrm{D}$ : 
(a) Ohmic plasmas in low triangularity shape with low fuelling rate of $1 \times 10^{21} \mathrm{Ds}^{-1}$. In 34 discharges $1764 \mathrm{Pam}^{3} \mathrm{D}$ had been injected with a fuel retention of $38 \mathrm{Pam}^{3}$ or equivalent $1.9 \times 10^{22} \mathrm{D}$ atoms. The retention rate is as low as $2.3 \times 10^{19} \mathrm{Ds}^{-1}$ when normalised to $807 \mathrm{~s}$ plasma operation in x-point configuration. The repetition rate between discharges is approximately 20 minutes with an overall of $14 h$ operation time for which the intershot outgassing is collected.

(b) L-mode plasmas with $P_{\text {aux }}=0.8 M W \mathrm{RF}$ heating in high triangularity shape with low fuelling rate. In 22 discharges $2266 \mathrm{Pam}^{3} \mathrm{D}$ had been injected with an integral fuel retention of $43 \mathrm{Pam}^{3}$ or equivalent $2.1 \times 10^{22} \mathrm{D}$ atoms. With the cumulated divertor duration of $440 \mathrm{~s}$, the retention rate amounts to $4.8 \times 10^{19} \mathrm{Ds}^{-1}$ compared with $1.27 \times 10^{21} \mathrm{Ds}^{-1}$ in the CFC reference under comparable conditions. In line with the reduction of the retention rate is also the reduction of the $\mathrm{C}$ impurity flux in the divertor by an order of magnitude. Low triangular plasmas in L-mode with comparable fuelling rate and divertor plasma conditions show comparable retention rates.

(c) In L-mode plasmas with $P_{a u x}=0.5 M W$ RF heating and with solely turbo molecular pumping 35 discharges have been carried out cumulating $731 \mathrm{~s}$ in divertor configuration. In total $1710 \mathrm{Pam}^{3} \mathrm{D}$ had been injected with a fuel retention of $222 \mathrm{Pam}^{3}$ or equivalent $11.1 \times 10^{22} \mathrm{D}$ atoms. The corresponding retention rate amounts to $1.51 \times 10^{20} \mathrm{Ds}^{-1}$ with an uncertainty of $\pm 1.8 \times 10^{19} \mathrm{Ds}^{-1}$.

(d) H-mode plasmas with $P_{a u x}=5.0 M W$ auxiliary heating by NBI and high fuelling rate
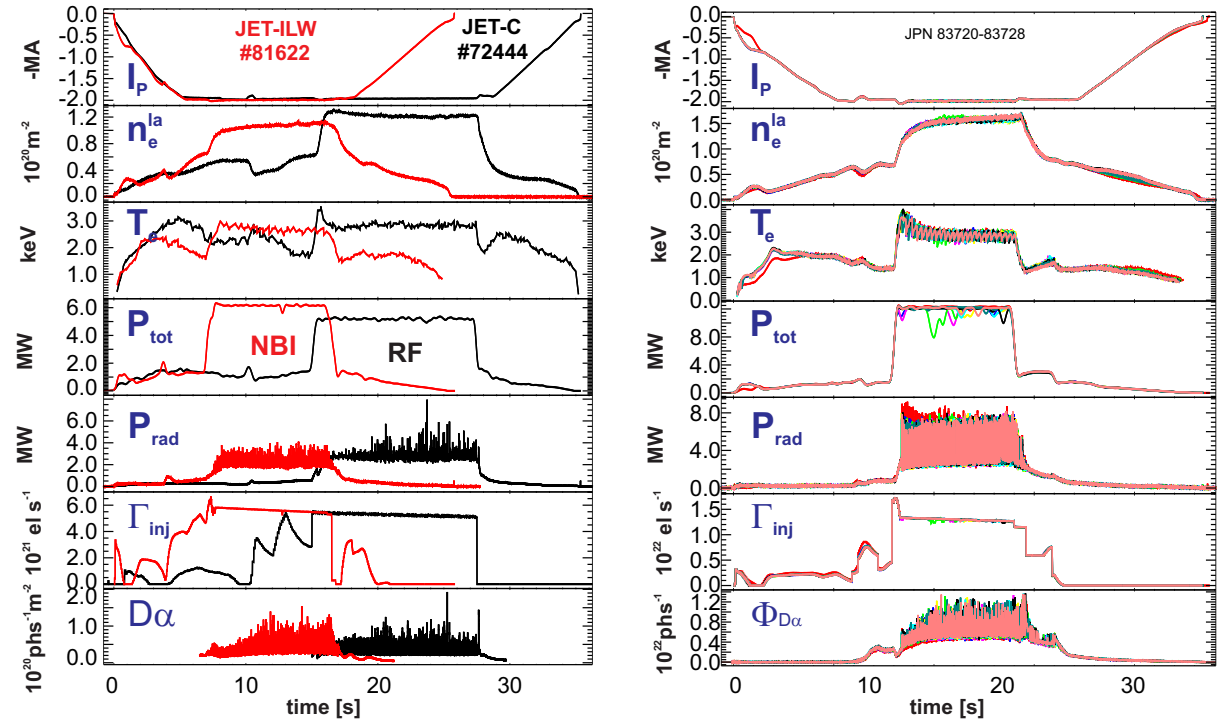

FIG. 2: a) Comparison of JET-C and JET-ILW discharges in type III ELMy H-mode (d). b) High reproducibility of 9 consecutive H-mode discharges with JET-ILW (cf $\boldsymbol{e}$ ).

$\left(6 \times 10^{21} D s^{-1}\right)$ in type III ELMy H-mode. In 18 discharges $2495 \mathrm{Pam}^{3}$ gas has been introduced by injection and beam fuelling, and $47 \mathrm{Pam}^{3}$ or $2.3 \times 10^{22} \mathrm{D}$ atoms were retained. The retention rate with the ILW amounts to $7.2 \times 10^{19} \mathrm{Ds}^{-1}$, when normalised to $317 \mathrm{~s}$ in x-point configuration, and can be compared with $1.37 \times 10^{21} D s^{-1}$ obtained in similar plasmas in CFC (fig.2a) but with RF heating of the same degree. Global plasma temperature, density and radiation as well as edge density and ion flux are in good agreement.

(e) As an example of a number of gas balance experiments in baseline H-mode plasmas which have been carried out throughout the campaign showing all a strong reduc- 
tion retention rates with respect to comparable JET-C plasmas (1), we present here in more detail one experiment from the last campaign before the shutdown for tile intervention. This specific campaign aimed in the provision of a material migration footprint in a characteristic ILW-typical H-mode plasma for the post mortem analysis of toroidal and poloidal sections of the first wall armour which will be executed in the next months. In order to accumulate a significant fluence, 151 identical discharges $\left(B_{t}=2.0 M A, I_{p}=2.0 M A, Z_{\text {eff }}=1.2, \delta=0.2, P_{\text {aux }}=12.0 \mathrm{MW}\right.$ NBI heating) in deuterium with in total 2500 plasma seconds in divertor configuration resulting in an integrated divertor fluence of $5.25 \times 10^{26} \mathrm{Dm}^{-2}$, comparable to one quarter of an ITER pulse $\left(2.5 \times 10^{27} D\right)$ at full performance [13], have been performed. To minimise the smearing of the erosion/deposition foot print caused by the impact of impinging deuterium ion and impurity flux onto the target plates, the limiter time was minimised to $1 / 3$ of the total pulse duration, the strike-point kept constant over the whole discharge, and the fuelling rate fixed to $1.4 \times 10^{22} \mathrm{Ds}^{-1}$. These parameters were chosen to ensure both strike points attached and to avoid at the same time potential $\mathrm{W}$ accumulation. The local plasma conditions at the inner target are $T_{e}=7 \mathrm{eV}$ and $n_{e}=2.5 \times 10^{20} \mathrm{~m}^{-3}$ and $T_{e}=35 \mathrm{~V}$ and $n_{e}=6 \times 10^{19} \mathrm{~m}^{-3}$ at the outer target plate. Operation with static magnetic shape (see fig. 1b) requires a cool-down phase of about 50 minutes between discharges to allow inertial cooling of the $\mathrm{W}$ divertor from max. $1050^{\circ} \mathrm{C}$ back to the base level of $160^{\circ} \mathrm{C}$. This allows significant outgassing over a period twice as long as in JET-C H-modes.

Three dedicated gas balances have been executed in this period to underline the reproducibility of gas balances under comparable conditions and to provide a reference for the fuel content deduced by post mortem analysis. Fig. $2 \mathrm{~b}$ shows the high reproducibility of plasma conditions in the series of 9 consecutive discharges of the second balance experiment marked as (e) in fig. 1a. The global parameters core electron density $n_{e}$ and temperature $T_{e}$, the input $P_{a u x}$ and radiated power $P_{r a d}$, as well as the recycling flux at the target, characterised by the $D_{\alpha}$ photon flux are similar throughout the whole series. These unseeded and purely NBI-heated discharges accumulated $151 \mathrm{~s}$ of plasma time in x-point configuration with a total divertor fluence of $3.2 \times 10^{25} \mathrm{Dm}^{2}$ and $2113 \mathrm{Pam}^{3}$ of injected D. A retention of $299 \mathrm{Pam}^{3} \mathrm{D}$ or equivalent $1.49 \times 10^{22} \mathrm{D}$ atoms was obtained under consideration of the pumping of outgassed D by the NBI cryogenic pumps during the time between discharges. This corresponds to a retention rate of $0.98 \times 10^{20} \mathrm{Ds}^{-1}$ as shown in fig.1a in the yellow bar labelled as (e). The red column represents the apparent rate assuming comparable outgassing time between discharges as performed in JET-C; this overestimated rate of $1.6 \times 10^{20} \mathrm{Ds}^{-1}$ can be compared with $1.97 \times 10^{21} \mathrm{Ds}^{-1}$ [6] measured for similar H-mode plasmas in JET-C. Overall this JET-ILW retention rate is in good agreement with the first gas balance of the same kind depicted in the presequential column as well as the first H-mode gas balance performed several months before, indicating comparable wall conditions over this experimental period.

\section{Discussion and Conclusion}

In carbon-dominated machines as previously in JET-C the fuel retention is governed by co-deposition of fuel in carbon layers on plasma wetted, and in remote and partially inaccessible areas. Short term retention and outgassing has been documented in experiments with carbon walls, but the magnitude was small with respect to the long term retention of typically a few $10^{21} D s^{-1}$ measured integral by global gas balance 1 hour after plasma 
operation. The long term retention rate dropped substantially with introduction of the full metallic wall in JET and the reduction of the $\mathrm{C}$ content by a factor 20 [9]. To estimate the physics mechanisms of the fuel retention under the new metallic wall conditions, information from post mortem tile analysis is required. But from present view the most likely mechanism for the remaining fuel retention in the JET-ILW experiments is most
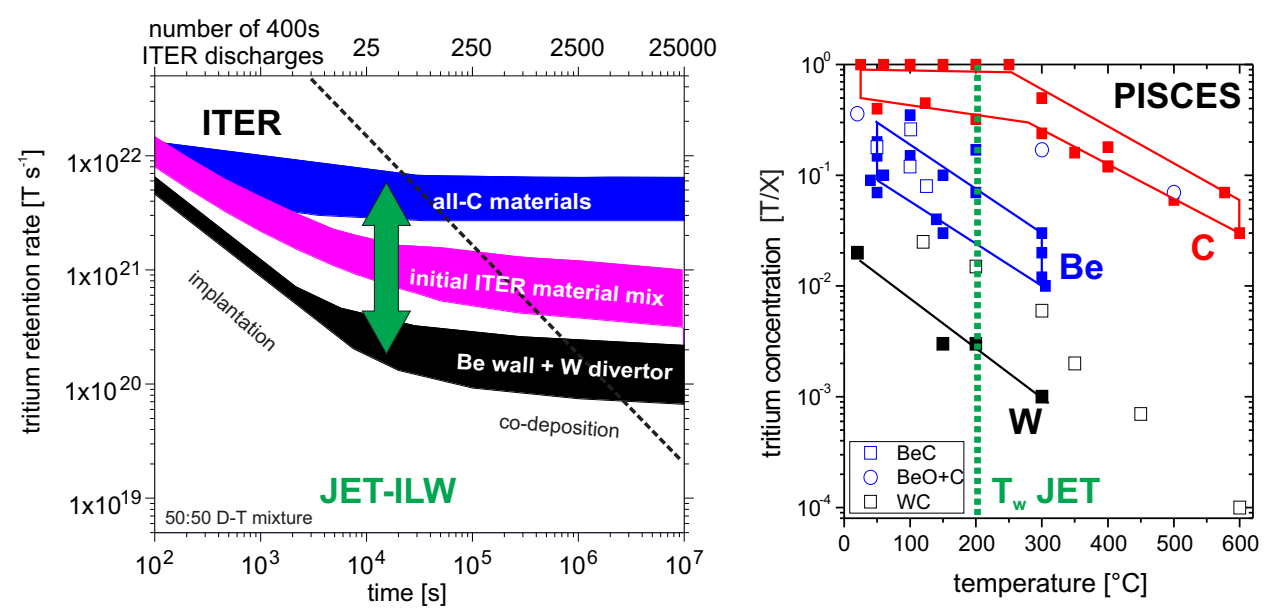

FIG. 3: a) ITER predictions for the retention rate and the accessible JET window. b) Fuel concentration in mixed layers [3] with the wall temperature of the JET-ILW indicated.

likely co-deposition of fuel in Be-layers which is in line with the measured high Be influx from the main chamber into the inner divertor leg whose plasma-facing surfaces are a net deposition zone. PISCES studies of fuel content in Be layers depicted in fig 3b showed a ten times lower $\mathrm{D}$ content than $\mathrm{C}$ layers at similar temperatures [3] which can explain the observed behaviour in JET with the Be main chamber wall. However, the surface morphology and the layer composition are of importance for the fuel content, too, and so far no information about the type of co-deposits in JET-ILW have been obtained. Co-deposition with $\mathrm{W}$ is not of importance and implantation is main mechanism for the retention in $\mathrm{W}$ as experiments in ASDEX Upgrade confirmed [15], but it plays itself a negligible role in comparison with the Be co-deposition.

Within the explored operational range from ohmic to H-mode plasmas, the long-term retention rate with the ILW exhibits a significant decrease by at least a factor 10 compared to carbon wall references leading to an upper limiter of $1.5 \times 10^{20} \mathrm{Ds}^{-1}$. Long-term outgassing, described for Be limiters in detail in [14], has also been observed in-situ in the post pulse operation of JET-ILW for about 100h with a total pressure and a partial pressure (mass $4 D_{2}$ ) decay according to $\propto t^{-0.8}$, and leads to further reduction of the fuel content in the metallic PFCs. Therefore it is expected, that the post mortem analysis of Be and $\mathrm{W}$ components will show a significant lower long term fuel content. The tiles will be retrieved from the vessel during the current vent end of 2012 and their analysis will provide information on the long term fuel content in co-deposited layers and in the bulk material by implantation - complementary to the gas balance studies reflecting a one day experiments presented here. The retention of the latter may be a factor 4 lower with respect to gas balances if the outgassing behaviour is comparable to CFC.

These results on fuel retention with the JET-ILW confirm qualitatively the predicted reduction of fuel retention in ITER with Be first wall and W divertor compared with a hypothetical full carbon ITER as shown in figure $3 \mathrm{a}$ and described in the comprehensive studies 
in [5]. It should be noted that no experimental comparison exists with a tokamak with Be walls and carbon divertor. In this figure the tritium retention rate, thus half the total retention rate of $\mathrm{D}$ and $\mathrm{T}$, is shown as function of operational time in full performance ITER 50:50 DT plasmas. In the case of the Be/W mix two phases can be distinguished in which the retention takes place; the fuel implantation in Be and $\mathrm{W}$ dominates at first followed by fuel co-deposition with Be. The first process saturates in time which is reflected in the reduction of the retention rate depicted in fig. 2a) whereas the almost constant rate in the later phase indicate the linear process of co-deposition which is qualitatively comparable to carbon - but more than one order of magnitude lower. Corresponding modelling on basis of JET-ILW results of the first year just started, however it can be expected that the modelled behaviour will be qualitative similar with the constraint that the implantationdominated part will be less pronounced due to the about four times smaller first wall area of JET in comparison to ITER. A semi-quantitative comparison of the measured JET-C retention rates, which are in the range of $1.0-3.0 \times 10^{21} \mathrm{Ds}^{-1}$, with the modelled values for a hypothetical full C ITER, which are in the range of $0.4-1.2 \times 10^{22} \mathrm{Ds}^{-1}$ at a cumulative operation time of $1 \times 10^{5} \mathrm{~s}$, close to the total operation time with JET-ILW of $0.7 \times 10^{5} s$, provides a factor 4 between JET and ITER. Taking this ratio and the reduction from JET-C to the JET-ILW with the maximum retention rate of $1.5 \times 10^{20} \mathrm{Ds}^{-1}$ into account, the $\mathrm{D}+\mathrm{T}$ retention rate for ITER with $\mathrm{Be} / \mathrm{W}$ can in a first attempt be predicted to $6.0 \times 10^{20} D s^{-1}$ for the phase where codeposition dominates. This is an upper limit as outgassing over nights and non-operational time is not considered here. Therefore, the number of ITER full performance discharges without active cleaning would amount about 1500 assuming the tritium inventory limit of $700 \mathrm{~g}$. This is a significant increase of the operational time without intervention for fuel removal in full plasma performance with respect to both a hypothetical CFC first wall and the currently baseline configuration with CFC at the strike-points. It is expected that modelling and post mortem analysis of tiles will increase further the confidence and precision in extrapolations to ITER.

This work, supported by the European Communities under the contract of Association between EURATOM/FZJ, was carried out within the framework of EFDA. The views and opinions expressed herein do not necessarily reflect those of the European Commission

\section{References}

[1] R.A. Pitts et al., J. Nucl. Mater. 415 S (2011) S957

[2] S. Brezinsek et al., J. Nucl. Mater 337339 (2005) 1058

[3] J. Roth et al., J. Nucl. Mater. 390-391 (2009) 1

[4] G.F. Matthews Phys. Scripta T145 (2012) 014001

[5] S. Brezinsek et al., J. Nucl. Mater. 415 S (2011) S936

[6] T. Loarer et al., J. Nucl. Mater. 390-391 (2009) 20

[7] V. Philipps et al., Fus. Eng. Des. 85 (2010) 1581

[8] G.F. Matthews presented at 20th PSI conference 2012

[9] S. Brezinsek presented at 20th PSI conference 2012

[10] R. Lässer et al. Fus. Eng. Des. 47 (1999) 173

[11] T. Loarer presented at 20th PSI conference 2012

[12] V. Philipps presented at 20th PSI conference 2012

[13] A. Kukushkin presented at 20th PSI conference 2012

[14] V. Philipps et al., J. Vac. Sci. Technol. A 112 (1993) 437

[15] M. Mayer et al., J. of Nucl. Mater. 390-391(2009) 538 